\title{
OPEN Relationship between CYP2D6 genotype, activity score and phenotype in a pediatric Thai population treated with risperidone
}

\author{
Yaowaluck Hongkaew ${ }^{1,2,3}$, Andrea Gaedigk ${ }^{4,5}$, Bob Wilffert ${ }^{6,7}$, Nattawat Ngamsamut ${ }^{8}$,
} Wiranpat Kittitharaphan ${ }^{8}$, Penkhae Limsila ${ }^{8}$ \& Chonlaphat Sukasem ${ }^{1,2 \varpi}$

Recently, the Clinical Pharmacogenetics Implementation Consortium (CPIC) have revised recommendations for the translation of CYP2D6 genotype to phenotype. Changes affect phenotype grouping, as well as the value used to calculate activity score for the $C Y P 2 D 6 * 10$ allele to better reflect the substantially decreased activity of this allele which is the most frequent allele found in Asian populations. This study aimed to evaluate whether the lower value for CYP2D6*10 as recommended, and the revised phenotype groupings improve the relationship between CYP2D6 genotype and risperidone measures. One hundred and ninety-nine children and adolescents with autism treated with a risperidone-based regimen for at least four weeks were included. CYP2D6 genotype was determined using the Luminex XTAG CYP2D6 Kit assay and translated into phenotype using different translation methods. Plasma concentrations of risperidone and 9-hydroxyrisperidone were measured using LC/MS/MS. Plasma levels of risperidone, risperidone concentration/dose ratio, and risperidone/9-hydroxyrisperidone ratio in patients with an activity score $<1$ were significantly higher than those $\geq 1$ ( $P$ value $<0.001$ for all three parameters). Plasma risperidone levels and risperidone concentration/dose ratios were significantly higher in intermediate metabolizers (defined as AS $=0.25-$ 0.75 ) than normal metabolizer (defined as AS $=1-2)$ patients $(1.44 \mathrm{vs} .0 .23 \mathrm{ng} / \mathrm{ml}, P<0.001$ and 1.63 vs. $0.29 \mathrm{ng} / \mathrm{ml} / \mathrm{ng}, P<0.001$, respectively) as well as risperidone/9-hydroxyrisperidone ratio ( $0.20 \mathrm{vs.}$ $0.04, P<0.001)$. This is the first study in an Asian population utilizing the revised CPIC-recommended method for translating the CYP2D6 genotype to phenotype. In addition to validating that CYP2D6 genetic variation significantly impacts risperidone metabolism, we demonstrated that revised value for the CYP2D6*10 was superior for genotype to phenotype translation. However, at least for risperidone, subjects with an activity score of 1 presented as phenotypic normal, and not intermediate metabolizers, suggesting that phenotype classification is substrate dependent.

Cytochrome P450 (P450) 2D6 is a major drug-metabolizing enzyme expressed in the liver ${ }^{1}$. CYP2D6 catalyzes the hepatic metabolism of a large number of clinically important medications, including codeine, amitriptyline, fluvoxamine, risperidone, fluoxetine, aripiprazole, paroxetine, and dextromethorphan ${ }^{23}$. The CYP2D6 gene is highly polymorphic. To date, over 130 allelic variants have been designated by the Pharmacogene Variation Consortium (PharmVar) ${ }^{4,5}$.

\footnotetext{
${ }^{1}$ Division of Pharmacogenomics and Personalized Medicine, Department of Pathology, Faculty of Medicine Ramathibodi Hospital, Mahidol University, Bangkok 10400, Thailand. 'Laboratory for Pharmacogenomics, Somdech Phra Debaratana Medical Center (SDMC), Ramathibodi Hospital, Bangkok, Thailand. ${ }^{3}$ Advanced Research and Development Laboratory, Bumrungrad International Hospital, Bangkok, Thailand. ${ }^{4}$ Division of Clinical Pharmacology, Toxicology and Therapeutic Innovation, Children's Mercy Kansas City, Kansas City, MO, USA. ${ }^{5}$ School of Medicine, University of Missouri-Kansas City, Kansas City, MO, USA. ${ }^{6}$ Unit of PharmacoTherapy, -Epidemiology and -Economics, Groningen Research Institute of Pharmacy, University of Groningen, Groningen, The Netherlands. ${ }^{7}$ Department of Clinical Pharmacy and Pharmacology, University of Groningen, University Medical Center Groningen, Groningen, The Netherlands. ${ }^{8}$ Yuwaprasart Waithayopathum Child and Adolescent Psychiatric Hospital, Department of Mental Health Services, Ministry of Public Health, Samut Prakan, Thailand. ${ }^{\square}$ email: chonlaphat.suk@mahidol.ac.th
} 
CYP2D6 allele frequencies vary substantially among different ethnic and ancestral populations ${ }^{6-9}$. The decreased function $C Y P 2 D 6^{*} 10$ allele $(100 \mathrm{C}>\mathrm{T}, \mathrm{P} 34 \mathrm{~S})$ is the most common allele in East Asian populations, including Thai, Chinese, Taiwanese, Korean, Vietnamese, and Filipino ${ }^{10-16}$. This allele is also observed in other populations, including Europeans, Africans, and their descendants, its frequency, however, considerably lower ${ }^{8}$. Conversely, the nonfunctional $C Y P 2 D 6^{*} 4$ allele is more frequent in European populations but is rarely observed in Asian populations ${ }^{8}$.

CYP2D6 genetic variation leads to a wide range of metabolic capacity ranging from no to increased activity. Based on their genotype, individuals are grouped into four phenotype groups, i.e., poor metabolizers (PMs), intermediate metabolizers (IMs), normal metabolizers (NMs), and ultrarapid metabolizers (UMs) ${ }^{17}$. The activity score system (AS) has been broadly accepted to translate the CYP2D6 genotype into phenotype and the Clinical Pharmacogenetics Implementation Consortium (CPIC) and the Dutch Pharmacogenetics Working Group (DPWG) for their respective guidelines ${ }^{18,19}$. Briefly, each allele is assigned a value of $0,0.5$ or 1 reflecting no function, decreased or normal function, and the sum of the values provides the AS of a genotype. The previous CPIC translation method classified $\mathrm{AS}=0$ as $\mathrm{PM}, \mathrm{AS}=0.5$ as $\mathrm{IM}, \mathrm{AS}=1$ to 2 as $\mathrm{NM}$, and $>2$ as UM. In an effort to harmonize genotype to phenotype translation, a CPIC-led working group has recently published a revised method and recommends using this new method to translate genotype to phenotype ${ }^{19}$. One major change was downgrading the value used for activity score calculation of the decreased function CYP2D $6^{\star} 10$ allele from 0.5 to 0.25 to more accurately reflect the dramatically decreased function of this allele. Furthermore, an AS of 1 is no longer categorized as NM, but as IM. While the new system has recently been applied to an in vitro study comprising mostly Caucasian liver tissue sample ${ }^{20}$, there are no investigations to date assessing the performance of the new method on any Asian populations with high frequencies of CYP2D6* 10 . There is also a paucity of information regarding the impact of substrate specificity on performance of the new translation method.

The use of a standardized method to infer phenotype from genotype is essential for test reporting and clinical implementation to prevent confusion and inconsistencies. We applied the new CPIC-recommended method to data obtained from risperidone (RIS)-treated Thai children and adolescents diagnosed with autism spectrum disorders (ASDs) and treated with RIS. Since the impact of CYP2D6 genotype on plasma concentrations of RIS is well-established ${ }^{21-25}$, RIS is a well-suited drug to evaluate whether the new translation method is superior over the previous method.

The aims of this investigation were to demonstrate whether the revised value for $C Y P 2 D 6^{*} 10$ indeed improves the relationship between AS and RIS plasma drug levels and to assess whether phenotype groupings, as recommended by CPIC, are appropriate for RIS.

\section{Subjects and methods}

Patients. One hundred and ninety-nine participants with ASD, aged 3-18 years, and diagnosed according to the Diagnostic and Statistical Manual of Mental Disorders, Fifth Edition (DSM-V) criteria in the Yuwaprasart Waithayopathum Child Psychiatric Hospital, Samut Prakan, Thailand, were recruited during 2017-2018. All patients were treated with a RIS-based regimen for at least four weeks before blood sample collection. Sociodemographic data were collected by a questionnaire including gender, age at assessment, daily RIS dosage, duration of RIS treatment, and concomitant medication. Patients were excluded if they were receiving concomitant treatments that could potentially affect RIS metabolism. This study was approved by the Ethics Review Committee on Human Research of the Faculty of Medicine Ramathibodi Hospital, Mahidol University, Thailand (MURA2017/556) and conducted in accordance with the Declaration of Helsinki. The study protocol was clearly explained to all participants and/or their legal guardians, and informed consent was given before the study.

Genotyping methods. Genomic DNA was extracted from EDTA blood with the MagNa Pure automated extraction system according to the manufacturer's instructions. A bead array platform genotyped CYP2D6 based on allele-specific primer extension (ASPE) and hybridization to oligonucleotide bound microspheres ${ }^{26}$ using the Luminex xTAG CYP2D6 Kit v3 (Luminex Corporation, Austin, TX, USA) according to the manufacturer's instructions ${ }^{27}$. The assay interrogates 21 variants including 19 CYP2D6 single nucleotide polymorphisms (SNPs): $-1584 \mathrm{C}>\mathrm{G}, 31 \mathrm{G}>\mathrm{A}, 100 \mathrm{C}>\mathrm{T}, 124 \mathrm{G}>\mathrm{A}, 137 \_138 \mathrm{insT}, 882 \mathrm{G}>\mathrm{C}, 1022 \mathrm{C}>\mathrm{T}, 1660 \mathrm{G}>\mathrm{A}, 1662 \mathrm{G}>\mathrm{C}$, 1708delT, 1759G $>$ T, 1847G $>$ A, 2550delA, 2616delAAG, 2851C $>$ T, 2936A $>$ C, 2989G $>$ A, 3184G $>$ A, and $4181 \mathrm{G}>\mathrm{C}$, as well as gene deletion and duplication $)^{25}$. The allelic variants called by this array are $C Y P 2 D 6^{\star} 1$ (assigned in the absence of variants; default assignment), ${ }^{\star} 2,{ }^{\star} 35$ (normal function), ${ }^{\star} 9,{ }^{\star} 10,{ }^{\star} 17,{ }^{\star} 29$ and ${ }^{\star} 41$ (decreased function), and $\star_{3},{ }^{*} 4,{ }^{\star} 5,{ }^{\star} 6,{ }^{*} 7,{ }^{\star} 8,{ }^{\star} 11$ and ${ }^{\star} 15$ (no function), as well as the presence of duplications. Patients who were carriers of a CYP2D6 duplication were excluded, because this array did not further characterize gene duplications (i.e. copy number or which allele is affected by the duplication). For instance, a duplication observed in an individual genotyped as $C Y P 2 D 6^{\star} 1{ }^{*} 10$ could result in e.g. a $C Y P 2 D 6^{\star} 1 x N /{ }^{*} 10$, CYP $2 D 6^{\star} 1 /{ }^{*} 10 x N$ or a ${ }^{\star} 1 /{ }^{\star} 36+{ }^{\star} 10$ genotype call.

To calculate the AS, values were assigned to the alleles identified in the study cohort as follows: no function alleles $\left({ }^{\star} 4,{ }^{\star} 5\right)=0$; the decreased function allele ${ }^{\star} 10=0.25$; other decreased function alleles $\left({ }^{\star} 14,{ }^{\star} 41\right)=0.5$, and normal function alleles $\left({ }^{\star} 1,{ }^{\star} 2,{ }^{\star} 35\right)=1$. The AS of each diplotype is the sum of the assigned value to each allele. Individuals with an AS of 0 were categorized as PMs, those with an AS of $0.25,0.5$ or 0.75 were categorized as IMs, and those with an AS of 1.25, 1.5, 1.75, or 2 were grouped as NMs. To compare translation methods, those with an AS of 1 were either categorized as IM (new CPIC method), or NM (previous CPIC method).

Analytical drug assay/plasma concentrations. Trough plasma concentration of RIS and its 9-OHRIS metabolite were quantified, between 8:00 and 10:00 AM, approximately $12 \mathrm{~h}$ after the bedtime dose, using a validated, previously published high-performance liquid chromatography procedure ${ }^{28}$. Briefly, we used an 


\begin{tabular}{|l|l|}
\hline Clinical information & Value \\
\hline Age (years); mean \pm SD & $9.25 \pm 3.93$ \\
\hline Male to female (M:F) ratio & $7: 1$ \\
\hline Daily risperidone dosage (mg/day); median (range), ng/ml & $0.75(0.10-5.00)$ \\
\hline Risperidone treatment duration (months); median (IQR), ng/ml & $43.47(16.40-76.60)$ \\
\hline Risperidone monotherapy, n (\%) & $118(59.30)$ \\
\hline Plasma drug levels, median (IQR), ng/ml & \\
\hline RIS level & $0.59(0.06-1.61)$ \\
\hline 9-OH-RIS level & $5.78(3.38-11.50)$ \\
\hline Active moiety level & $7.06(4.26-12.89)$ \\
\hline Ratio of risperidone/9-OH-RIS & $0.08(0.02-0.24)$ \\
\hline Plasma concentration-to-dose (C/D) ratios, median (IQR), ng/ml/mg & \\
\hline C/D of RIS & $0.71(0.17-2.25)$ \\
\hline C/D of 9-OH-RIS & $8.45(5.34-12.65)$ \\
\hline C/D of the active moiety & $9.60(6.20-15.76)$ \\
\hline
\end{tabular}

Table 1. Patient demographics $(\mathrm{n}=199)$. RIS risperidone, 9-OH-RIS 9-hydroxyrisperidone, Active moiety, the sum of risperidone plus 9-OH-RIS, $C / D$ dose-corrected concentration, $S D$ standard deviation, IQR interquartile range.

Agilent 1260 HPLC system (Agilent Technologies, CA, USA), which was connected to an AB Sciex API 3200 (Applied Biosystems, Foster City, CA, USA) instrument. Chromatographic separation was achieved on the C18 column $(4.6 \mathrm{~cm} \times 50 \mathrm{~mm} ; 1.8 \mathrm{~mm}$ particle size). Integration of peak areas and determination of the concentrations was performed with the Analyst 1.5.2 software (Applied Biosystems, CA, USA). Quadratic regression with $1 / \times$ weighted concentrations was used. The mean inter- and intra-assay accuracy for both RIS and 9-OH-RIS was set within $\pm 15.0 \%$ Relative Error of nominal, and precision $<15.0 \%$ Relative Standard Deviation.

Statistical analysis. Descriptive statistics were used to describe the clinical characteristics of the subjects. Data were expressed as mean (standard deviation, SD) or median (interquartile range, IQR) in normal or nonnormal distribution data, respectively. The nonparametric Kruskal-Wallis (comparisons more than two groups) and Mann-Whitney $U$ tests (comparisons between two groups) were used to assess the association between plasma drug levels and the studied genotypes or predicted phenotypes at each time point. Statistical analyses were carried out using SPSS v24 (SPSS Inc., Chicago, IL, USA) for Windows. Statistical significance is reported as $P<0.05$ for a two-tailed distribution.

\section{Results}

Demographic and clinical characteristics. Our sample consisted of 199 children and adolescents with a mean age of 9.25 (SD; 3.93) years who had been diagnosed with autism spectrum disorders. Demographic data are presented in Table 1. Participants were treated with a RIS-based regimen. One hundred and eighteen patients (59.3\%) received RIS monotherapy. The medications that were concomitantly prescribed to patients were methylphenidate, sodium valproic acid, benzhexol, topiramate, cetirizine, clonazepam, hypodine, phenytoin, and phenobarbital. There were no significant differences for RIS or 9-OH-RIS between children and adolescents. Most of which were male (174; 87.44\%). There were also no significant differences for RIS or 9-OH-RIS between males and females nor those receiving monotherapy and polytherapy.

Distribution of the CYP2D6 alleles and genotypes. The CYP2D6*10 decreased function allele was the most common allele identified among the 199 subjects at $51.8 \%$. The frequencies of the normal function alleles $C Y P 2 D 6^{\star} 1$ and $C Y P 2 D 6^{\star} 2$ were $25.1 \%$ and $6.3 \%$, respectively. Another decreased function allele, CYP2D6 ${ }^{\star} 41$, was observed at $6.8 \%$. CYP2D6 $6^{\star} 4$ and $C Y P 2 D 6^{\star} 5$, both nonfunctional alleles, were found at frequencies of $1.3 \%$ and $8.3 \%$, respectively. We also observed two subjects with the rare CYP2D6 $6^{\star} 14$ allele $(0.50 \%)$ in this study cohort. CYP2D6 allele frequencies are presented in Table 2. Of the 398 alleles, 125 were normal function (aggregate frequency of 31.4\%) and were assigned a value of 1 to calculate the AS while 29 decreased function alleles (aggregate frequency of $7.3 \%$ ) received a value of 0.5 and 38 no function alleles (aggregate frequency of $9.6 \%$ ) received a value of 0 .

Genotype frequencies are summarized in Supplementary Table 1. Of the 20 CYP2D6 genotypes identified, CYP $2 D 6^{*} 1{ }^{*} 10$ was the most frequent $(29.6 \%)$, followed by $C Y P 2 D 6^{*} 10 /{ }^{*} 10, C Y P 2 D 6^{\star} 5 /{ }^{*} 10$, and $C Y P 2 D 6^{*} 10 /{ }^{*} 41$ $(26.1 \%, 7.5 \%$, and $7.5 \%$, respectively).

Plasma levels and C/D of RIS, 9-OH-RIS, active moiety, and RIS/9-OH-RIS ratio in the different CYP2D6 AS groups. The relationship between CYP2D6 AS, RIS plasma concentration, and the 9-OH-RIS metabolite was examined in 199 patients (Table 3). Patients were divided into eight groups (AS of $0,0.25,0.5,0.75,1,1.25,1.5$, and 2$)$. The most common AS was $1.25(35.18 \%)$, comprising $C Y P 2 D 6^{*} 1{ }^{*} 10$ and CYP2D $6^{*} 2 /{ }^{*} 10$ genotypes. There were significant differences in RIS, the metabolic ratio RIS/9-OH-RIS, and C/D 


\begin{tabular}{|l|l|l|}
\hline Alleles & CPIC clinical function & Frequency (\%) \\
\hline${ }^{*} 1$ & Normal function & $100(25.1 \%)$ \\
\hline${ }^{* 2}$ & Normal function & $25(6.3 \%)$ \\
\hline${ }^{*} 4$ & No function & $5(1.3 \%)$ \\
\hline${ }^{*} 5$ & No function & $33(8.3 \%)$ \\
\hline${ }^{*} 10$ & Decreased function & $206(51.8 \%)$ \\
\hline${ }^{*} 14$ & Decreased function & $2(0.5 \%)$ \\
\hline${ }^{*} 41$ & Decreased function & $27(6.8 \%)$ \\
\hline
\end{tabular}

Table 2. CYP2D6 allele frequencies $(\mathrm{n}=199)$. Allele definitions are per PharmVar at https://www.pharmvar. org/gene/CYP2D6.

\begin{tabular}{|c|c|c|c|c|c|c|c|c|}
\hline AS & n (\%) & RIS (ng/ml) & 9-OH-RIS (ng/ml) & $\begin{array}{l}\text { Active moiety (ng/ } \\
\text { ml) }\end{array}$ & $\begin{array}{l}\text { Ratio of RIS/9- } \\
\text { OH-RIS }\end{array}$ & $\begin{array}{l}\text { C/D of RIS (ng/ } \\
\mathrm{ml} / \mathrm{mg} \text { ) }\end{array}$ & $\begin{array}{l}\text { C/D of 9-OH-RIS } \\
(\mathrm{ng} / \mathrm{ml} / \mathrm{mg})\end{array}$ & $\begin{array}{l}\text { C/D of active } \\
\text { moiety }(\mathrm{ng} / \mathrm{ml} / \\
\mathrm{mg})\end{array}$ \\
\hline $\mathrm{AS}=0$ & $1(0.50)$ & 2.67 & 1.78 & 4.45 & 1.50 & 10.68 & 7.12 & 17.80 \\
\hline $\mathrm{AS}=0.25$ & $17(8.54)$ & $1.43(0.68-4.20) \mathrm{a}$ & $5.11(3.86-13.56)$ & $8.17(4.53-22.95)$ & $0.35(0.17-0.82) \mathrm{a}$ & $1.45(0.82-4.77) \mathrm{a}$ & $5.50(3.86-8.00) \mathrm{d}$ & $9.01(5.47-16.34)$ \\
\hline $\mathrm{AS}=0.5$ & $55(27.64)$ & $1.10(0.35-2.54) \mathrm{a}$ & $5.32(3.11-11.40)$ & $6.11(4.54-11.96)$ & $0.19(0.06-0.35) \mathrm{a}$ & $1.48(0.34-2.73) \mathrm{a}$ & $8.13(5.42-11.93)$ & $10.23(6.32-14.39)$ \\
\hline $\mathrm{AS}=0.75$ & $15(7.54)$ & $1.61(0.92-2.58) \mathrm{a}$ & $5.13(3.78-7.45)$ & $7.25(5.28-9.76)$ & $0.26(0.19-0.35) \mathrm{a}$ & $2.24(1.52-3.71) \mathrm{a}$ & $10.15(6.33-16.00)$ & $11.68(7.83-19.87)$ \\
\hline $\mathrm{AS}=1.0$ & $17(8.54)$ & $0.44(0.05-1.20)$ & $7.56(2.71-11.63)$ & $7.56(2.79-12.83)$ & $0.05(0.02-0.11)$ & $0.27(0.19-2.40)$ & $10.90(6.68-21.40)$ & $11.79(6.81-21.48)$ \\
\hline $\mathrm{AS}=1.25$ & $70(35.18)$ & $0.33(0.05-0.74)$ & $7.27(4.30-11.23)$ & $7.60(4.31-12.78)$ & $0.04(0.02-0.08)$ & $0.36(0.15-0.99)$ & $9.18(6.74-14.13)$ & $9.72(6.74-15.05)$ \\
\hline $\mathrm{AS}=1.5$ & $9(4.52)$ & $0.13(0.05-0.49)$ & $2.28(1.44-9.75)$ & $4.19(1.44-9.88)$ & $0.05(0.04-0.07)$ & $0.40(0.10-0.61)$ & $6.74(2.88-8.45)$ & $8.38(2.88-9.06)$ \\
\hline $\mathrm{AS}=2.0$ & $15(7.54)$ & $0.05(0.02-0.27)$ & $8.36(6.36-12.87)$ & $8.36(6.71-13.61)$ & $0.01(0.00-0.03)$ & $0.05(0.03-0.49)$ & $7.95(5.28-19.45)$ & $8.08(5.33-21.13)$ \\
\hline
\end{tabular}

Table 3. Plasma levels and C/D of RIS, 9-OH-RIS, active moiety, and RIS/9-OH-RIS ratio among CYP2D6

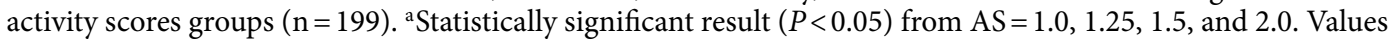
expressed as median (interquartile range). AS activity score (assigned per revised CPIC recommendations), $C / D$ dose-corrected concentration, RIS Risperidone, 9-OH-RIS 9-hydroxy-risperidone, Active moiety, the sum of risperidone plus 9-OH-RIS.

of RIS plasma concentrations between AS of $0.25,0.5,0.75$, and $1,1.25,1.5,2$. There was a significant difference between patients when divided into two groups, one with $\mathrm{AS}<1$ and the other with $\mathrm{AS} \geq 1$. Plasma levels of RIS and RIS/9-OH-RIS ratio, and plasma C/D of RIS in patients with $\mathrm{AS}<1$ were significantly higher than those in patients with $\mathrm{AS} \geq 1$ ( $P$ value $<0.001$ among three drug parameters) (Fig. 1A-C). When genotypes with an AS of 1 were categorized as IM, significance of RIS, RIS/9-OH-RIS ratio, and RIS C/D between AS of 1 and AS $>1$ was considerably lower as reflected by a $P$ value of $0.412,0.519$, and 0.314 , compared to a $P$ value of $0.005,0.000$, and 0.015 between AS of 1 and AS $<1$. Based on these findings, individuals with an AS of 1 presented as NMs rather than IMs, while all others fit within their respective phenotype categories.

Association between plasma RIS parameters and predicted phenotypes. Based on the above findings, patients with an AS of 0, AS of 0.25-0.75, and AS of 1-2 presented as, and were thus classified, as PM, IM, and NM, respectively. Fifty-six percentages of patients $(n=111)$ were NMs, followed by IMs $(n=87,43.7 \%)$. There was only one patient with a predicted PM phenotype of $0.5 \%$.

There were statistically significant differences for the plasma RIS concentration $(P<0.001)$ and RIS/9-OH-RIS ratio $(P<0.001)$ when subjects were categorized as described above (Table 4 and Fig. 1$)$. The plasma concentration of RIS among IMs (AS $=0.25-0.75,1.44 \mathrm{ng} / \mathrm{ml}$ ) was significantly higher compared to that among NMs (AS $=1-2,0.25 \mathrm{ng} / \mathrm{ml}, P<0.001)$ and lower when compared to that found in the PM individual $(2.67 \mathrm{ng} / \mathrm{ml})$. The RIS/9-OH-RIS ratio in IM subjects was statistically significantly higher than the ratio observed in the NMs (AS $=1-2,0.20$ vs. $0.04, P<0.001)$. These patients also had a significantly higher C/D of RIS than NMs $(1.63$ vs. $0.29 \mathrm{ng} / \mathrm{ml} / \mathrm{mg}, P<0.001)$.

\section{Discussion}

To the best of our knowledge, this is the first study applying the revised CPIC recommendations for the translation of CYP2D6 genotype to phenotype in an Asian population. This new method is anticipated to have a considerable impact on Asians compared to other populations due to the high frequency of the CYP $2 D 6^{\star} 10$ allele. This allele conveys a considerable decrease in function and thus was downgraded, i.e., now receives a lower value for AS calculation, to improve the accuracy of phenotype prediction. The CPIC recommendations are drug-agnostic, i.e., the phenotype does not take substrate-specificity into account. Thus, in addition to evaluating whether the revised value for CYP2D6*10 improves the relationship between RIS, RIS/9-OH-RIS ratio, and C/D of RIS, we also assessed whether phenotype groupings, as recommended by CPIC, are appropriate for RIS. 

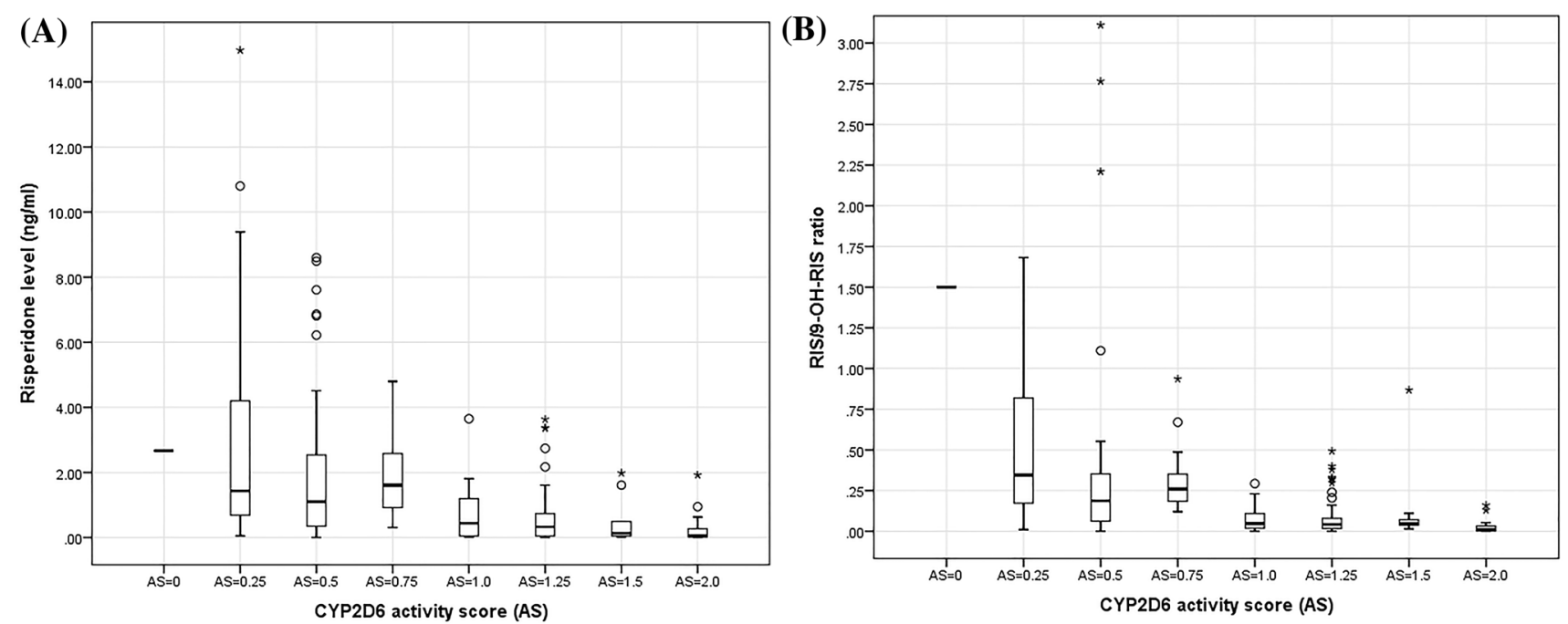

(C)

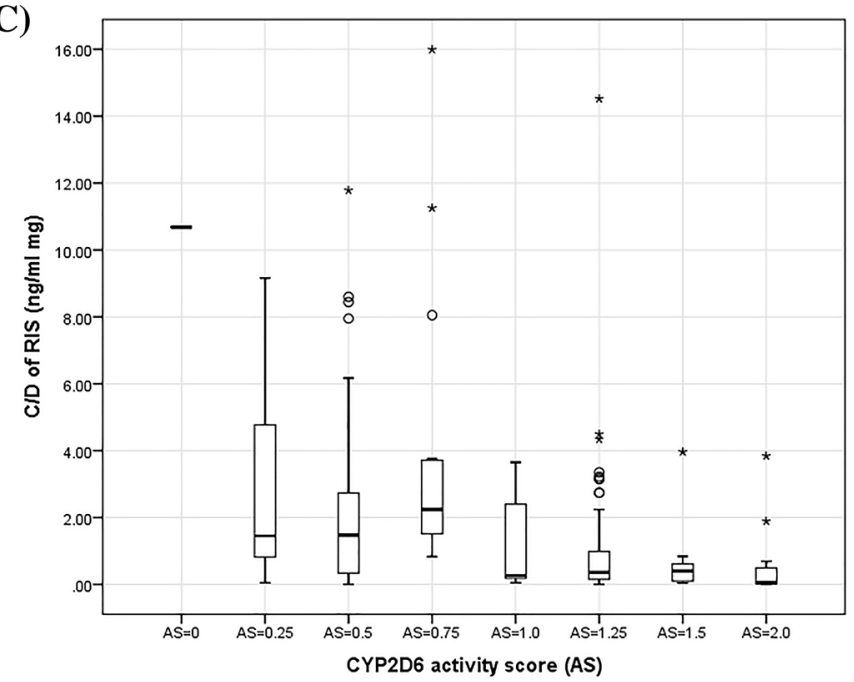

Figure 1. Plasma levels of RIS (A), RIS/9-OH-RIS ratio (B), and C/D of RIS (C) among activity score (AS) groups. AS was calculated using a value of 0.25 for the decreased function CYP $2 D 6^{\star} 10$ allele.

\begin{tabular}{|l|l|l|l|l|l|l|l|l|}
\hline $\begin{array}{l}\text { CYP2D6 predicted } \\
\text { phenotype }\end{array}$ & $\mathbf{n}(\mathbf{\%})$ & RIS (ng/ml) & 9-OH-RIS (ng/ml) & $\begin{array}{l}\text { Active moiety } \\
(\mathbf{n g} / \mathbf{m l})\end{array}$ & $\begin{array}{l}\text { Ratio of RIS/9- } \\
\text { OH-RIS }\end{array}$ & $\begin{array}{l}\text { C/D of RIS (ng/ } \\
\mathbf{m l} / \mathbf{m g})\end{array}$ & $\begin{array}{l}\text { C/D of 9-OH-RIS } \\
\mathbf{( n g / m l} / \mathbf{m g})\end{array}$ & $\begin{array}{l}\text { C/D of active } \\
\mathbf{m o i e t y}(\mathbf{n g} / \mathbf{m l} / \\
\mathbf{m g})\end{array}$ \\
\hline PM & $1(0.5)$ & 2.67 & 1.78 & 4.45 & 1.50 & 10.68 & 7.12 & 17.80 \\
\hline IM & $87(43.7)$ & $1.44(0.65-2.95)$ & $5.22(3.57-11.40)$ & $6.50(4.54-14.40)$ & $0.20(0.13-0.37)$ & $1.63(0.83-3.66)$ & $7.74(5.24-11.40)$ & $9.85(6.20-15.34)$ \\
\hline NM & $111(55.8)$ & $0.25(0.05-0.74)$ & $7.33(3.68-11.67)$ & $7.88(4.01-12.89)$ & $0.04(0.01-0.08)$ & $0.29(0.09-0.93)$ & $9.03(6.28-14.13)$ & $9.50(6.43-16.04)$ \\
\hline$P$ value $^{\mathrm{a}}$ & & $<0.001^{\mathrm{a}}$ & 0.185 & 0.836 & $<0.001^{\mathrm{a}}$ & $<0.001^{\mathrm{a}}$ & 0.105 & 0.879 \\
\hline
\end{tabular}

Table 4. Plasma levels and C/D ratios of RIS, 9-OH-RIS, active moiety, and RIS/9-OH-RIS ratio among

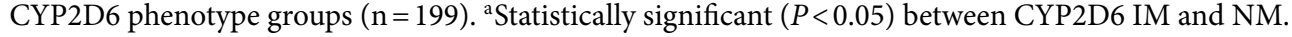
CYP2D6 PM, AS =0; IM, AS = 0.25, 0.5, and 0.75; NM, AS = 1.0, 1.25, 1.5 and 2.0. PM poor metabolizer, $I M$ intermediate metabolizer, $N M$ normal metabolizer, $C / D$ dose-corrected concentration, RIS Risperidone, 9-OH-RIS 9-hydroxy-risperidone, Active moiety, the sum of RIS plus 9-OH-RIS.

Owing to the revised AS definition, a notable number of subjects would be reclassified as IMs (Fig. 2). Specifically, 17 subjects with an AS of 1 which were grouped as NM under the old method would be grouped as IMs under the new method. Their observed phenotype, however, identified them as NMs suggesting that the recommended classification system does not improve phenotype prediction for RIS. In contrast, using the lower value of 0.25 for $C Y P 2 D 6^{*} 10$ AS calculation did improve the relationship between AS and RIS, RIS/9-OH-RIS ratio, and C/D of RIS. Similar findings were observed by Brown et al. who showed that systemic exposure of atomoxetine $(\mathrm{AUC}-\infty)$ of AS of 1 was not significantly different from that observed for subjects with an AS of 


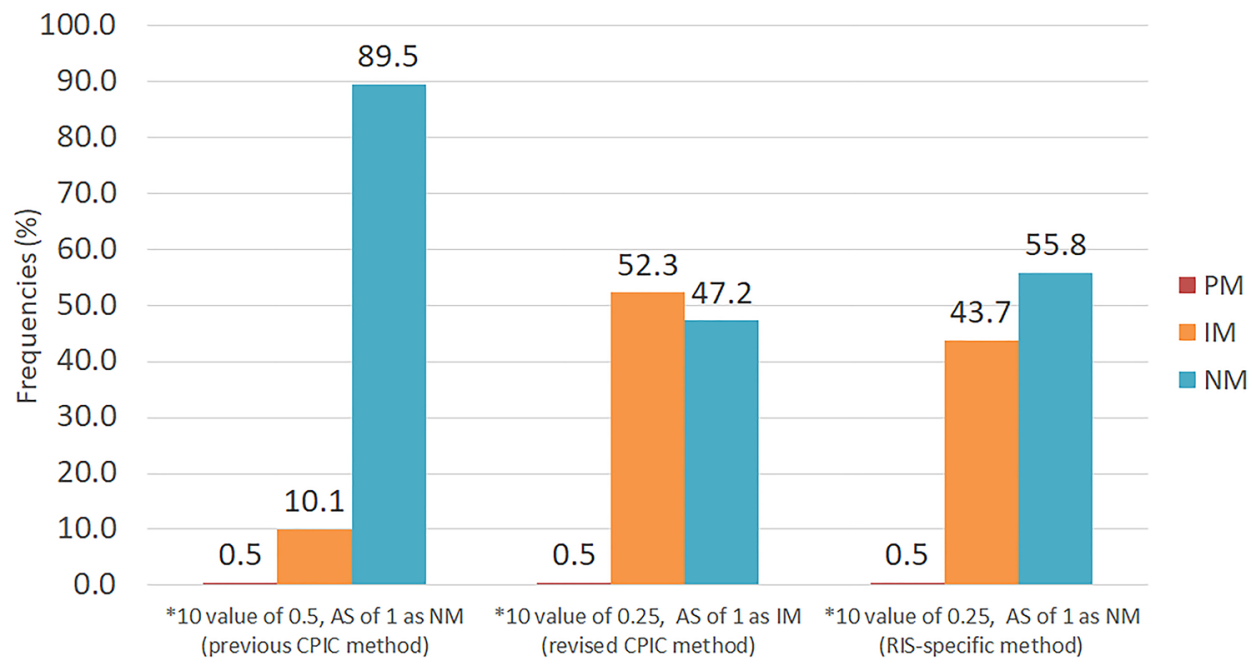

Figure 2. Frequencies of each predicted phenotypes using different genotype to phenotype translation methods. The previous CPIC method assigned CYP2D6* 10 a value of 0.5 and classified subjects with an AS of 1 as NMs while the revised CPIC method assigns a value of 0.25 to the CYP2D $6^{\star} 10$ allele and classifies subjects with an AS of 1 as IMs. This study used a value of 0.25 for $C Y P 2 D 6^{\star} 10$ and classified subjects with an AS of 1 as NMs to predict phenotype for RIS. PM, poor metabolizer; IM, intermediate metabolizer; NM, normal metabolizer.

\begin{tabular}{|l|l|l|l|l|}
\hline CYP2D6 predicted phenotype & $\begin{array}{l}\text { Previous DPWG activity score } \\
\text { definition }\end{array}$ & $\begin{array}{l}\text { Previous CPIC activity score } \\
\text { definition }\end{array}$ & $\begin{array}{l}\text { Revised CPIC and DPWG activity } \\
\text { score definition }\end{array}$ & This study (Thai autism cohort) \\
\hline Ultrarapid metabolizer (UM) & $>2.5$ & $>2$ & $>2.25$ & N/A \\
\hline Normal metabolizer (NM) & $1.5-2.5$ & $1-2$ & $1.25-2.25$ & $1-2$ \\
\hline Intermediate metabolizer (IM) & $0.5-1$ & 0.5 & $0.25-1$ & $0.25-0.75$ \\
\hline Poor metabolizer (PM) & 0 & 0 & 0 & 0 \\
\hline
\end{tabular}

Table 5. Comparisons of CYP2D6 predicted phenotypes translation according to CYP2D6 activity score. N/A not available.

1.5 or $2^{29}$. In addition, Frederiksen et $\mathrm{al}^{30}$, demonstrated allele-specific metabolism of vortioxetine suggesting substantial differences among decreased function allele. Taken together, these findings raise awareness of the limitations and pitfalls of drug-agnostic genotype to phenotype translation methods. This is further substantiated by the plasma concentrations of RIS and RIS/9-OH-RIS ratios being significantly higher in AS of $0.25-0.75$ than AS of 1-2 arguing that the former should be classified as IMs and the latter as NMs. Therefore, to predict CYP2D6 phenotype for RIS treatment, genotype should be translated into phenotype as shown in Table 5.

Additionally, the CYP2D6 genotype (or AS) had a substantial impact on the trough dose-corrected plasma concentration of RIS. In accordance with results we previously reported for a different cohort, there were statistically significant differences in the plasma concentration for RIS $(P<0.001)$ and the RIS/9-OH-RIS ratio $(P<0.001)$ among phenotype groups in Thai autism children ${ }^{25,31}$. Furthermore, PM patients had significantly higher RIS C/D than those genotyped as $C Y P 2 D 6^{*} 1{ }^{*} 1^{32}$. The same pattern was also observed in another study ${ }^{33}$, i.e., the C/D ratio for RIS was significantly different in CYP2D6 PMs. The presence of the CYP2D6*10 allele was also associated with significantly higher levels of C/D of RIS levels at week $12(P=0.003)$ in North Indian patients with schizophrenia ${ }^{34}$. Moreover, plasma RIS/9-OH-RIS ratios were significantly higher in patients with an AS of 0.5 compared to those with an AS of 2 in an independent cohort of Thai subjects ${ }^{24}$. Taken together, the RIS/9-OH-RIS metabolic ratio is a biomarker for CYP2D6 activity, which may be useful to guide the treatment of patients in need of psychotropic drugs ${ }^{35}$.

There were no significant differences in 9-OH-RIS and total active moiety concentrations among the CYP2D6 predicted phenotype groups, as found in an earlier study ${ }^{32}$. Similarly, the total active moiety, sum of the plasma concentrations of RIS and 9-OH-RIS, corrected for the dose, did not significantly differ between individuals of different genotypes. These findings are consistent with a previous study in another Thai cohort of ASD patients $s^{25,31}$ that showed no significant differences in 9-OH-RIS and active moiety concentrations. This finding is consistent with a previous study using positron emission tomography scans of healthy volunteers after receiving a single oral dose of RIS showing that plasma concentrations of the sum of RIS and 9-OH-RIS partly overlapped between the $\mathrm{NMs}$ and $\mathrm{PMs}^{36}$. Therefore, the plasma concentrations of the 9-OH-RIS and total active moiety are independent of the CYP2D6-related metabolism. It has been suggested that the efflux transporter ABCB1, as well as CYP3A5 can contribute to the steady-state plasma concentration of RIS, 9-OH-RIS, and active moiety ${ }^{37,38}$. 
As mentioned above, the CPIC-recommended drug-agnostic method to predict phenotype may not accurately predict phenotype across all drugs and all allelic variants. Regardless of the imperfections and shortcomings of the method, using a standardized system, although imperfect, is preferable because it makes comparisons of results among studies easier. However, it also demonstrates the need to develop more sophisticated algorithms that take substrate specificity, among other patient-specific information, into account.

We acknowledge the following limitations of the Luminex platform. This test does not quantitatively determine copy number nor does it determine which allele is duplicated or identify any other structural variants. Furthermore, only the most common alleles are tested. We speculate that some subjects may have rare or novel alleles which may explain some of the outliers shown in Fig. 1. In conclusion, the new CPIC recommended genotype to phenotype translation method, developed to promote standardized phenotype classification has its limitations for RIS. Using AS, rather than phenotype may be more accurate for this drug, especially considering the broad range of CYP2D6 activity and substrate specify. The findings of our study provide valuable information to further the implementation of genotype-guided risperidone treatment.

Received: 13 October 2020; Accepted: 4 February 2021

Published online: 18 February 2021

\section{References}

1. Pelkonen, O. \& Raunio, H. Metabolic activation of toxins: Tissue-specific expression and metabolism in target organs. Environ. Health Perspect. 105, 767-774 (1997).

2. Pan, X., Ning, M. \& Jeong, H. Transcriptional regulation of CYP2D6 expression. Drug Metab. Dispos. 45, 42-48 (2017).

3. Saravanakumar, A., Sadighi, A., Ryu, R. \& Akhlaghi, F. Physicochemical properties, biotransformation, and transport pathways of established and newly approved medications: A systematic review of the top 200 most prescribed drugs vs. the FDA-approved drugs between 2005 and 2016. Clin. Pharmacokinet. 58, 1281-1294 (2019).

4. Gaedigk, A. et al. The pharmacogene variation (PharmVar) consortium: Incorporation of the human cytochrome P450 (CYP) allele nomenclature database. Clin. Pharmacol. Ther. 103, 399-401 (2018).

5. Nofziger, C. et al. PharmVar GeneFocus: CYP2D6. Clin. Pharmacol. Ther. 107, 154-170 (2020).

6. Del Tredici, A. L. et al. Frequency of CYP2D6 alleles including structural variants in the United States. Front. Pharmacol. 9, 305 (2018).

7. Qiao, W. et al. Integrated CYP2D6 interrogation for multiethnic copy number and tandem allele detection. Pharmacogenomics 20, 9-20 (2019).

8. Gaedigk, A., Sangkuhl, K., Whirl-Carrillo, M., Klein, T. \& Leeder, J. S. Prediction of CYP2D6 phenotype from genotype across world populations. Genet Med. 19, 69-76 (2017).

9. Gene-specific Information Tables for CYP2D6. January 29, 2019. www.pharmgkb.org/page/cyp2d6RefMaterials.

10. Dorji, P. W., Tshering, G. \& Na-Bangchang, K. CYP2C9, CYP2C19, CYP2D6 and CYP3A5 polymorphisms in South-East and East Asian populations: A systematic review. J. Clin. Pharm. Ther. 44, 508-524 (2019).

11. Chamnanphon, M. et al. CYP2D6 genotype analysis of a Thai population: Platform comparison. Pharmacogenomics 19, 947-960 (2018).

12. Nguyen, H. H. et al. Single nucleotide and structural variants of CYP2D6 gene in Kinh Vietnamese population. Medicine (Baltimore) 98, e15891 (2019).

13. Baclig, M. O. et al. Allelic and genotype frequencies of catechol-O-methyltransferase (Val158Met) and CYP2D6*10 (Pro34Ser) single nucleotide polymorphisms in the Philippines. Int. J. Mol. Epidemiol. Genet. 3, 115-121 (2012).

14. Liou, Y. H., Lin, C. T., Wu, Y. J. \& Wu, L. S. The high prevalence of the poor and ultrarapid metabolite alleles of CYP2D6, CYP2C9, CYP2C19, CYP3A4, and CYP3A5 in Taiwanese population. J. Hum. Genet. 51, 857 (2006).

15. Byeon, J. Y. et al. CYP2D6 allele frequencies in Korean population, comparison with East Asian, Caucasian and African populations, and the comparison of metabolic activity of CYP2D6 genotypes. Arch. Pharm. Res. 41, 921-930 (2018).

16. Chen, R., Zheng, X. \& Hu, P. CYP2D6 phenotyping using urine, plasma, and saliva metabolic ratios to assess the impact of CYP2D6 $\left.{ }^{*}\right) 10$ on interindividual variation in a Chinese population. Front. Pharmacol. 8, 239 (2017).

17. Gaedigk, A. Complexities of CYP2D6 gene analysis and interpretation. Int. Rev. Psychiatry. 25, 534-553 (2013).

18. Caudle, K. E. et al. Standardizing CYP2D6 genotype to phenotype translation: Consensus recommendations from the clinical pharmacogenetics implementation consortium and dutch pharmacogenetics working group. Clin. Transl. Sci. 13, 116-124 (2020).

19. CYP2D6 Genotype to Phenotype Standardization Project. 5th October 2019. https://cpicpgx.org/resources/cyp2d6-genotype-tophenotype-standardization-project/.

20. Dalton, R. et al. Interrogation of CYP2D6 structural variant alleles improves the correlation between CYP2D6 genotype and CYP2D6-mediated metabolic activity. Clin. Transl. Sci. 13, 147-156 (2020).

21. Berecz, R. et al. Relationship between risperidone and 9-hydroxy-risperidone plasma concentrations and CYP2D6 enzyme activity in psychiatric patients. Pharmacopsychiatry 35, 231-234 (2002).

22. Roh, H. K. et al. Risperidone metabolism in relation to CYP2D6*10 allele in Korean schizophrenic patients. Eur. J. Clin. Pharmacol. 57, 671-675 (2001).

23. Scordo, M. G. et al. Cytochrome P450 2D6 genotype and steady state plasma levels of risperidone and 9-hydroxyrisperidone. Psychopharmacology 147, 300-305 (1999).

24. Vanwong, N. et al. Impact of CYP2D6 polymorphism on steady-state plasma levels of risperidone and 9-hydroxyrisperidone in Thai children and adolescents with autism spectrum disorder. J. Child. Adolesc. Psychopharmacol 27, 185-191 (2017).

25. Vanwong, N. et al. Detection of CYP2D6 polymorphism using Luminex xTAG technology in autism spectrum disorder: CYP2D6 activity score and its association with risperidone levels. Drug Metab. Pharmacokinet. 31, 156-162 (2016).

26. Lyon, E. et al. Laboratory testing of CYP2D6 alleles in relation to tamoxifen therapy. Genet. Med. 14, 990-1000 (2012).

27. Ruano, G. et al. Practical interpretation of CYP2D6 haplotypes: Comparison and integration of automated and expert calling. Clin. Chim. Acta. 456, 7-14 (2016).

28. Vanwong, N. et al. Development and validation of liquid chromatography/tandem mass spectrometry analysis for therapeutic drug monitoring of risperidone and 9-hydroxyrisperidone in pediatric patients with autism spectrum disorders. J. Clin. Lab. Anal. 30, $1236-1246$ (2016).

29. Brown, J. T. et al. Single dose, CYP2D6 genotype-stratified pharmacokinetic study of atomoxetine in children with ADHD. Clin. Pharmacol. Ther. 99, 642-650 (2016).

30. Frederiksen, T., Areberg, J., Schmidt, E., Bjerregaard Stage, T. \& Brosen, K. Quantification of in vivo metabolic activity of CYP2D6 genotypes and alleles through population pharmacokinetic analysis of vortioxetine. Clin. Pharmacol. Ther. 109, 150-159 (2020). 
31. Nuntamool, N. et al. Pharmacogenomics and efficacy of risperidone long-term treatment in Thai autistic children and adolescents. Basic Clin. Pharmacol. Toxicol. 121, 316-324 (2017).

32. Jovanovic, N. et al. The role of CYP2D6 and ABCB1 pharmacogenetics in drug-naive patients with first-episode schizophrenia treated with risperidone. Eur. J. Clin. Pharmacol. 66, 1109-1117 (2010).

33. Patteet, L. et al. Erratum to: Genotype and co-medication dependent CYP2D6 metabolic activity: Effects on serum concentrations of aripiprazole, haloperidol, risperidone, paliperidone and zuclopenthixol. Eur. J. Clin. Pharmacol. 73, 127 (2017).

34. Chavan, B. S., Kaur, G., Gupta, D. \& Aneja, J. A prospective study to evaluate the effect of CYP2D6 polymorphism on plasma level of risperidone and its metabolite in north indian patients with schizophrenia. Indian J. Psychol. Med. 40, 335-342 (2018).

35. Mannheimer, B. et al. Risperidone metabolic ratio as a biomarker of individual CYP2D6 genotype in schizophrenic patients. Eur. J. Clin. Pharmacol. 70, 695-699 (2014).

36. Nyberg, S., Dahl, M. L. \& Halldin, C. A PET study of D2 and 5-HT2 receptor occupancy induced by risperidone in poor metabolizers of debrisoquin and risperidone. Psychopharmacology 119, 345-348 (1995).

37. Kang, R. H. et al. Effects of CYP2D6 and CYP3A5 genotypes on the plasma concentrations of risperidone and 9-hydroxyrisperidone in Korean schizophrenic patients. J. Clin. Psychopharmacol. 29, 272-277 (2009).

38. Xiang, Q., Zhao, X., Zhou, Y., Duan, J. L. \& Cui, Y. M. Effect of CYP2D6, CYP3A5, and MDR1 genetic polymorphisms on the pharmacokinetics of risperidone and its active moiety. J. Clin. Pharmacol. 50, 659-666 (2010).

\title{
Acknowledgements
}

Financial support from the Thailand Research Fund through the Royal Golden Jubilee Ph.D. Program (Grant No. PHD/0107/2557) to Yaowaluck Hongkaew and Chonlaphat Sukasem is acknowledged. The authors thank all the staff in Yuwaprasart Waithayopathum Child and Adolescent Psychiatric Hospital and all the children and adolescents with ASD who participated in the study.

\section{Author contributions}

Y.H. wrote the manuscript; N.N., P.L., and C.S. designed the research; W.K., N.N., and P.L. collected the samples and data; Y.H. performed the research; Y.H. analyzed the data. Y.H., A.G., B.W., and C.S. revised the critical revision of the article; A.G., and C.S. approved the final version to be published.

\section{Funding}

This study was supported by grants of the (1) the Thailand Research Fund through the Royal Golden Jubilee Ph.D. Program (Grant No. PHD/0107/2557), (2) Pharmacogenomics for Autistic Child Project, Khoon Poom Foundation, The Project of Her Royal Highness Princess Ubonratana Rajakanya Siriwatana Bhanawadee, (3) Office of National Research Council of Thailand (4) Faculty of Medicine Ramathibodi Hospital and (5) Mahidol University.

\section{Competing interests}

The authors do not declare affiliations or financial involvement with any organization or entity with a financial interest in or financial conflict with the subject matter or materials discussed in the manuscript.

\section{Additional information}

Supplementary Information The online version contains supplementary material available at https://doi. org/10.1038/s41598-021-83570-w.

Correspondence and requests for materials should be addressed to C.S.

Reprints and permissions information is available at www.nature.com/reprints.

Publisher's note Springer Nature remains neutral with regard to jurisdictional claims in published maps and institutional affiliations.

Open Access This article is licensed under a Creative Commons Attribution 4.0 International License, which permits use, sharing, adaptation, distribution and reproduction in any medium or format, as long as you give appropriate credit to the original author(s) and the source, provide a link to the Creative Commons licence, and indicate if changes were made. The images or other third party material in this article are included in the article's Creative Commons licence, unless indicated otherwise in a credit line to the material. If material is not included in the article's Creative Commons licence and your intended use is not permitted by statutory regulation or exceeds the permitted use, you will need to obtain permission directly from the copyright holder. To view a copy of this licence, visit http://creativecommons.org/licenses/by/4.0/.
\end{abstract}

(C) The Author(s) 2021 